\title{
Alteration of Introns in a Hyaluronan Synthase 1 (HAS1) Minigene Convert Pre-Mrna Splicing to the Aberrant Pattern in Multiple Myeloma (MM): MM Patients Harbor Similar Changes
}

\author{
Jitra Kriangkum*, Amanda Warkinton, Andrew R. Belch, Linda M. Pilarski* \\ Department of Oncology, Cross Cancer Institute, University of Alberta, Edmonton, Alberta, Canada
}

\begin{abstract}
Aberrant pre-mRNA splice variants of hyaluronan synthase 1 (HAS1) have been identified in malignant cells from cancer patients. Bioinformatic analysis suggests that intronic sequence changes can underlie aberrant splicing. Deletions and mutations were introduced into HAS1 minigene constructs to identify regions that can influence aberrant intronic splicing, comparing the splicing pattern in transfectants with that in multiple myeloma (MM) patients. Introduced genetic variations in introns 3 and 4 of HAS1 as shown here can promote aberrant splicing of the type detected in malignant cells from MM patients. HAS1Vd is a novel intronic splice variant first identified here. HAS1Vb, an intronic splice variant previously identified in patients, skips exon 4 and utilizes the same intron 4 alternative 3 'splice site as HAS1Vd. For transfected constructs with unaltered introns 3 and 4, HAS1Vd transcripts are readily detectable, frequently to the exclusion of HAS1Vb. In contrast, in MM patients, HAS1Vb is more frequent than HAS1Vd. In the HAS1 minigene, combining deletion in intron 4 with mutations in intron 3 leads to a shift from HAS1Vd expression to HAS1Vb expression. The upregulation of aberrant splicing, exemplified here by the expression of HAS1Vb, is shown here to be influenced by multiple genetic changes in intronic sequences. For HAS1Vb, this includes enhanced exon 4 skipping and increased usage of alternative $3^{\prime}$ splice sites. Thus, the combination of introduced mutations in HAS1 intron3 with introduced deletions in HAS1 intron 4 promoted a shift to an aberrant splicing pattern previously shown to be clinically significant. Most MM patients harbor genetic variations in intron 4, and as shown here, nearly half harbor recurrent mutations in HAS1 intron 3. Our work suggests that aberrant intronic HAS1 splicing in MM patients may rely on intronic HAS1 deletions and mutations that are frequent in MM patients but absent from healthy donors.
\end{abstract}

Citation: Kriangkum J, Warkinton A, Belch AR, Pilarski LM (2013) Alteration of Introns in a Hyaluronan Synthase 1 (HAS1) Minigene Convert Pre-Mrna Splicing to the Aberrant Pattern in Multiple Myeloma (MM): MM Patients Harbor Similar Changes. PLoS ONE 8(1): e53469. doi:10.1371/journal.pone.0053469

Editor: Pal Bela Szecsi, Gentofte University Hospital, Denmark

Received September 7, 2012; Accepted November 30, 2012; Published January 3, 2013

Copyright: (c) 2013 Kriangkum et al. This is an open-access article distributed under the terms of the Creative Commons Attribution License, which permits unrestricted use, distribution, and reproduction in any medium, provided the original author and source are credited.

Funding: This work is funded by Alberta Cancer Foundation and Canadian Institutes of Health Research. The funders had no role in study design, data collection and analysis, decision to publish, or preparation of the manuscripts.

Competing Interests: The authors have declared that no competing interests exist.

*E-mail: jitra@ualberta.ca (JK); Ipilarsk@ualberta.ca (LMP)

\section{Introduction}

It is becoming increasingly apparent that splicing defects play a key role in cancer, and that genomic changes in splicing elements [1-3], sometimes termed "splicing spoilers" [4,5], can promote aberrant splicing. Because regulation of splicing is such a complex network $[1,4]$, all genetic variations in genomic DNA and premRNA should be evaluated for their impact on splicing within any given genomic context. It has been estimated that $\sim 50 \%$ of mutations underlying genetic diseases cause aberrant splicing [6]. Alterations in a splicing site or splicing control region can have long range implications for splicing events, including altered 3-D architecture of pre-mRNA, activation of cryptic splice sites, exclusion of exons and/or inclusion of all or part of introns. Single mutations can strengthen otherwise weak splice sites and discriminate against otherwise strong splice sites [2-4]. Defective mRNA splicing caused by single nucleotide polymorphisms (SNPs) and/or splice site mutations often results in inactivation of tumor suppressor activity (e.g. HRPT2 [7,8], PTEN [9], MLHI [10-12], ATR [13]) or generation of dominant negative inhibitors (e.g.
CHEK2 [14], VWOX [15]). In breast cancer, aberrantly spliced forms of progesterone and estrogen receptors are found (reviewed in [2]). Intronic mutations inactivate p53 through aberrant splicing and intron retention, leading to the production of no or inactive p53 [16]. The large number of silent p53 genetic variations in cancer tend to be non-randomly located in exonic splicing enhancers, with a likely impact on p53 splicing [17], perhaps explaining their selection during oncogenesis and indicating that so-called "silent" mutations can have a profound impact on function.

In MM patients, we have identified a series of aberrant splice variants $(\mathrm{Va}, \mathrm{Vb}$ and $\mathrm{Vc})$ in the hyaluronan synthase 1 gene $[18,19]$. These splice variants were found only in MM B cells, being undetectable in B cells from healthy donors. Alternative splicing of HASl involves exon 4 skipping ( $\mathrm{Va}$ ), partial intron 4 retention $(\mathrm{Vc})$ or a combination of both $(\mathrm{Vb})$. HAS $1 \mathrm{Vb}$ expression correlates with significantly reduced survival in a cohort of MM patients [19]. Functional analysis of HAS1 minigene in transfectants shows that aberrant HAS1 splice variants gain anchorage 
independence and are transforming in vivo [20]. To determine the genetic basis for aberrant HAS1 splicing, the HAS1 gene from multiple cell types was sequenced for multiple patients, leading to identification of multiple exonic and intronic mutations, as well as SNPs, insertions/deletions and substitutions [21]. Although absent from healthy donors, a proportion of the newly identified HAS1 mutations were independently acquired in multiple unrelated patients, termed "recurrent". Bioinformatic analysis predicts that a combination of novel mutations, SNPs and insertions/deletions in HASl direct the aberrant splicing that correlates with poor outcome [21], supporting the clinical relevance of genetic variations that lead to aberrant HAS1 splicing. However, splicing is a complex process and there are likely to be many combinations of genetic changes that can lead to aberrant splicing of HAS1.

In the present studies, we have introduced deletions and mutations into HASl constructs to identify some of the regions that influence aberrant intronic splicing, comparing the splicing patterns obtained in transfectants with those we detect in patients with MM. We find that introduced genetic variations in HAS1 constructs convert in vitro splicing patterns to the patterns seen in vivo in patients. We also find that genomic DNA from MM patients harbors novel recurrent mutations in HAS1 introns that appear to regulate aberrant splicing in transfectants. Our work suggests that aberrant intronic HASl splicing in MM patients relies on intronic HASl mutations that are frequent in $\mathrm{MM}$ patients but absent from healthy donors.

\section{Materials and Methods}

\section{Ethics Statement}

The study was approved by the Ethics Committee of the Cross Cancer Institute and University of Alberta. Written informed consent was provided in accordance with the Declaration of Helsinki.

\section{Plasmid Construction}

HAS1FL (FLc) and HAS1g345 (G345) have been previously described [20,21]. In brief, FLc is generated by cloning of HAS1 cDNA fragment into a mammalian expression vector pcDNA3 (Invitrogen). G345 is generated by replacing exons 3-4-5 cDNA sequence in FLc with the corresponding genomic DNA fragment.

Deletion constructs del5, del4, del3, del2 and dell are derivatives of G345, being created by overlap extension PCR [22]. Two DNA subfragments were separately amplified: a) the 5' piece extending from the beginning of the G345 construct to $680 \mathrm{bp}$ downstream of exon 4 , and $\mathrm{b}$ ) the $3^{\prime}$ piece extending from the selected sequence in intron 4 to the end of the G345 construct. Overlapping ends were created by primer design. Joining of fragments was performed by mixing equimolar ratio of DNA fragments in standard polymerase chain reaction (PCR) using $\mathrm{HiFi}$ Taq_DNA polymerase (Invitrogen) in the absence of primers and run for 7 cycles at $94^{\circ} \mathrm{C}$ for $30 \mathrm{~s}$ and $72^{\circ} \mathrm{C}$ for $4 \mathrm{~min}$. The assembled fragment was further amplified in the presence of forward and reverse primers for 30 cycles, and then cloned into pcDNA3. The end products are constructs that have selective internal intron 4 deletion, each carried $680 \mathrm{bp}$ of upstream intronic sequence joined to a specified downstream intronic sequence. These are 489 bp (del5), 361 (del4), 263 bp (del3), $198 \mathrm{bp}(\mathrm{del} 2)$ and $84 \mathrm{bp}$ (dell) sequences upstream of exon 5 . The deleted protions are calculated to be 983 bp, 1111 bp, 1209 bp, $1274 \mathrm{bp}$ and 1388 bp respectively.

Mutagenized HAS1 intron 3 (G1-28 m) was custom made by minigene synthesis (Mr.Gene). Constructs G345/G1-28 m and dell/Gl-28 $\mathrm{m}$ were generated by overlap extension PCR of selected DNA fragments. For constructs carrying G1-18 m, G1928 m, G19-24 m, G25-28 m or G27-28 m, DNA subfragments were amplified either from the parental construct or from the G1$28 \mathrm{~m}$ derivative, then joined together by overlap extension PCR. The accuracy of each construct was validated by DNA sequencing.

\section{Transient Expression and HAS1 Splicing Analysis}

HeLa were originally obtained from the American Type Culture Collection (ATCC, Manassas, VA) and were grown at $37^{\circ} \mathrm{C}, 5 \% \mathrm{CO}_{2}$ in DMEM (Invitrogen) supplemented with $10 \%$ fetal bovine serum (Invitrogen). Transfection was performed using Lipofectamine 2000 (Invitrogen) according to the manufacturer's instructions. Twenty-four hours post-transfection, cells were washed twice with PBS and total RNA was prepared by Trizol reagent (Invitrogen) according to the manufacturer's instructions. cDNA was reversed transcribed using dT15 and Superscript II (Invitrogen). PCR was run for 30 cycles at $94^{\circ} \mathrm{C}$ for $30 \mathrm{~s}, 60^{\circ} \mathrm{C}$ for $30 \mathrm{~s}$, and $72^{\circ} \mathrm{C}$ for $30 \mathrm{~s}$ using Platinum Taq DNA polymerase (Invitrogen). PGR products were analyzed by agarose gel electrophoresis or DNA fragment analysis. For DNA fragment analysis, fluorescence primer was used and products were mixed with size standard in formamide and analyzed on an ABI Prism 3130xl Genetic Analyzer (Applied Biosystems). Data analysis was performed using GeneMapper software version 4.0. Primer sequences are summarized in Table 1.

\section{Analysis of HAS1Vb/Vd Expression in Peripheral Blood Mononuclear Cells (PBMC)}

Peripheral blood samples were collected from normal individuals and MM patients at diagnosis. MM was identified based on consensus criteria. Normal blood was obtained from University of Alberta Hospital emergency room as anonymous samples from 102 individuals selected as being over the age of 50 and without any obvious hematological issues.

PBMC were isolated by step gradient centrifugation (FicollPaque Plus; GE Healthcare). RT-PGR followed Transient expression and HAS1 splicing analysis section, except that amplification was run for 35 cycles using E3/E5I4 primer set (Table 1) and PCR products were analyzed by DNA fragment analysis.

\section{Analysis of Recurrent Mutations in HAS1 Intron 3}

Genomic DNA was prepared from PBMC using Trizol reagent (Invitrogen). HASl intron 3 region was amplified from $50 \mathrm{ng}$ genomic DNA using $5^{\prime}$ outer SNPs $/ 3^{\prime}$ exon 4 primer set at $94^{\circ} \mathrm{C}$ for

Table 1. Summary of primer sequences.

\begin{tabular}{|c|c|c|}
\hline Primer & Orientation & Sequence \\
\hline E3 & sense & 5'GGGCTTGTCAGAGCTACT T3' \\
\hline E5 & antisense & 5'AGGGCGTCTCTGAGTAGCAG 3' \\
\hline E514 & antisense & 5'CTGGAGGTGTACCTGCACGGGGGC3' \\
\hline 5'Vb-specific & sense & 5'GCGGTCCTCTAGAATCCTGCCCAG3' \\
\hline 5'outer SNPs & sense & 5'TGTTCAGATCGGTTGCAGAGT3' \\
\hline 3'exon 4 & antisense & 5'CATGCACACACGCTAGGATA3' \\
\hline HAS1seq5' & sense & 5'GGGGTCTGTGCTGATCCTGG3' \\
\hline HAS1seq3' & antisense & 5'AACTGCTGCAAGAGGTTATTCC3' \\
\hline$\beta 2 m$ & sense & 5'CCAGCAGAGAATGGAAAGTC3' \\
\hline$\beta 2 \mathrm{~m}$ & antisense & 5'GATGCTGCTTACATGTCTCG3' \\
\hline
\end{tabular}


$30 \mathrm{~s}, 60^{\circ} \mathrm{C}$ for $30 \mathrm{~s}$, and $72^{\circ} \mathrm{C}$ for $30 \mathrm{~s}$ for 35 cycles. The amplicon was treated with ExoSAP-IT reagent (USB) to remove excess primers and deoxyribonucleotides and subjected to direct sequencing using HASlseq5' or HAS1seq3' primer and BigDye Terminator v3.1 (Applied Biosystems). Sequencing reaction was run on an ABI Prism 3130xl Genetic Analyzer (Applied Biosystems) and data were analyzed by DNA Sequencing Analysis Software v5.1 and SeqScape Software v2.5. Primer sequences are summarized in Table 1. Polymerase error rate in this study is shown to be less than 1 in $14,000 \mathrm{bp}$.

\section{Results}

\section{In vitro Analysis of Minigene Construct Characterizes Alternative Splicing of Human HAS1}

HAS1 splicing analysis has been established in a mammalian expression system where splicing products can be assessed by RTPCR. Transient expression driven by the HAS1 minigene G345 construct (Figure 1A) yielded mainly full-length transcripts (FL) and varieties of alternatively spliced products similar to those found in ex vivo analysis [19] including a newly identified isoform termed HAS1Vd. Among HASl splice variants, Va (exon 4 skipped) is the most abundant, being detected along with FL on agarose gel when E3/E5 primer set was used (Figure 1B). Other variants are best determined by DNA fragment analysis using a selective primer set. HAS1Vb (exon 4 skipped and 59 bp downstream intron 4 retained) is of most interest due to its relevance in MM patients. Amplification by E3/E5I4 primer set predictably detected only $\mathrm{HAS} 1 \mathrm{Vb}$ as E5I4 primer binds to exon 5 /intron 4 junction. However, we always found another isoform, termed HAS1Vd, co-amplified with $\mathrm{HAS} 1 \mathrm{Vb}$, suggesting it is a common spliced product that has not been reported in the clinical studies (Figure 1C). Sequencing analysis showed that both $\mathrm{Vb}$ and $\mathrm{Vd}$ utilized the same alternative ${ }^{\prime}$ SS that retained $59 \mathrm{bp}$ of downstream intron $4(-59)$ : these two variants differed only in the inclusion $(\mathrm{Vd})$ or exclusion $(\mathrm{Vb})$ of exon 4 (133 bp). Overall, the splicing profile of G345 mimics normal HAS1 splicing and thus provides a model to study intronic sequence manipulation of the human HAS1 minigene.

\section{Unlike HAS1Vb, the Expression of HAS1Vd is Comparable in HD or MM PBMC}

Since HAS1Vd has not previously been reported, we evaluated its expression in PBMC of 102 healthy donors (HDs) and $93 \mathrm{MM}$ patients. Using E3/E5I4 primer set in RT-PCR and DNA fragment analysis, we found that $9 \%$ of both populations expressed HASlVd, suggesting that HASlVd has little clinical relevance (Supplementary Tables S1 and S2). However HAS1Vb, documented previously as having clinical relevance, was found in $20 \%$ of unfractionated MM PBMC compared to 5\% in HD PBMC, consistent with previous results [19]. Thus, MM PBMCs expressed $\mathrm{HASlVb}$ more frequent than $\mathrm{Vd}$ but $\mathrm{HD}$ PBMCs and transfectants expressed HASlVd more frequent than $\mathrm{Vb}$, indicating that for the variants analyzed, splicing directed by the G345 construct is similar to that of HD and differs from that occurring in MM patients.

\section{Partial Deletion of Intron 4 Increases Expression of HAS1Vd but not of HAS1Vb}

Increased $\mathrm{HAS} 1 \mathrm{Vb}$ was found to correlate with patient outcome in MM [19]. In MM and Waldenstrom's macroglobulinemia $(\mathrm{WM})$, we have identified recurrent mutations in HAS1 intron 4 [21,23]. In silico analysis predicts that mutations and

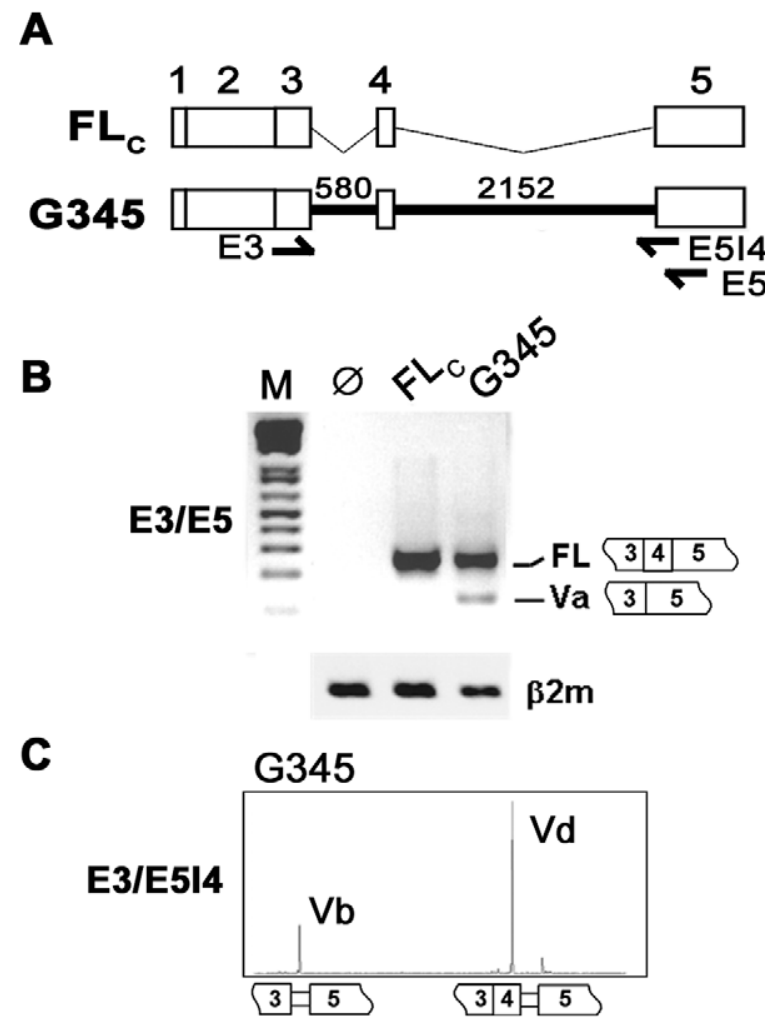

Figure 1. In vitro splicing analysis of human HAS1 minigene. Constructs FLC and G345 are shown in (A). Arrows show where PCR primers bind (E3, E5 and E5/4). The length of each intron in G345 is shown in bp. Each construct was transfected into HeLa cells and HAS1 splicing was studied by RT-PCR. Using E3/E5 primer set, products were analyzed by agarose gel electrophoresis (B). For E3/E5/4 primer set, amplicons were analyzed by DNA fragment analysis (C). Splice junctions for each product are also illustrated. $\varnothing$, mock transfection; $\beta 2 \mathrm{~m}$, control. doi:10.1371/journal.pone.0053469.g001

deletions in intron 4 can influence alternative splicing to use splice sites that generate HAS1Vb [21]. In this study, we determined if partial deletion of intron 4 is able to alter the splicing profile in vitro. A series of deletion constructs (del5-dell) was generated from G345, as mapped in Figure 2A. Deletion begins after $680 \mathrm{bp}$ downstream of $5^{\prime} \mathrm{SS}$ and ends at variable distance upstream of 3'SS. Spliced isoforms produced by transfectants were characterized by RT-PCR on agarose gel electrophoresis and confirmed by DNA fragment analysis and sequencing of subclones. Figure 2B showed that expression driven by del5, del4, del3 and del2 were comparable to that of parental G345. Deletion beyond del 2 encouraged the use of alternative 3'SS (-59) since increased HASIVd was observed in dell. Thus, intronic sequence $198 \mathrm{bp}$ upstream of exon 5 that is present in del2 is minimally required to mimic the splicing profile of the undeleted HAS1 minigene. This implies that the selected $1274 \mathrm{bp}$ internal sequence could potentially be dispensable. In contrast, expression of $\mathrm{HAS} 1 \mathrm{Vb}$ remains the same in all of the dell transfectants (Figure 2B), suggesting that changes in intron 4 on its own, in the absence of enhanced exon 4 skipping, is not sufficient to promote aberrant HAS $1 \mathrm{Vb}$ expression.

\section{Mutagenesis of G-repeat Motifs in Intron 3 Enhances Exon 4 Skipping}

The sequence of HAS1 intron $3(580 \mathrm{bp})$ is quite striking, comprising 28 repetitions of the motif (A/U)GGG (Figure 3). In 
A

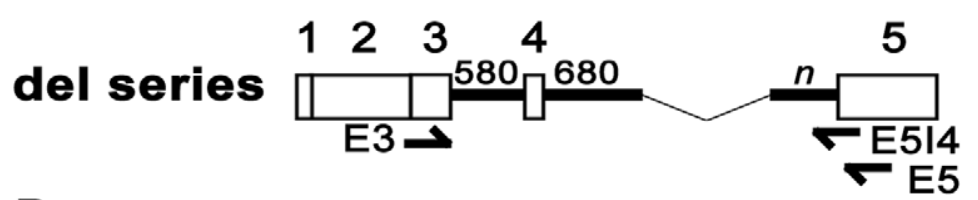

B
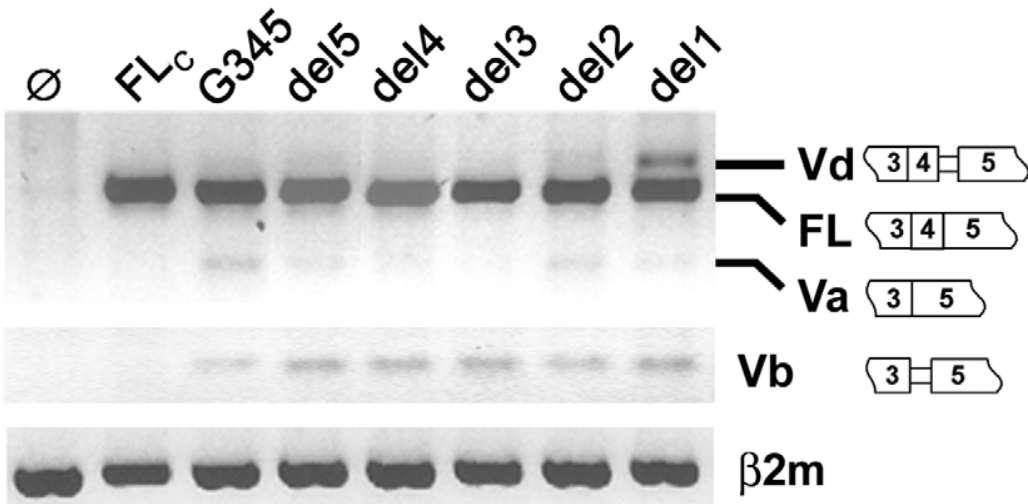

Figure 2. Partial deletion of intron 4 enhanced expression of HAS1Vd but not HAS1 Vb. Selective portions of intron 4 were removed from G345 to create a series of del constructs as illustrated in (A). Each del construct carried 680 bp sequence of upstream intron 4 joined to the selective sequence in the downstream intron 4 ( $n$ ). For del5, $n=489$ bp; del4, $n=361$ bp; del3, $n=263$ bp; del2, $n=198$ bp; del1, $n=84$ bp. Arrows show where PCR primers bind ( $5^{\prime} \mathrm{Vb}$ primer is not shown in this diagram). Splicing of HAS1 in HeLa transfectants is shown in (B). RT-PCR was amplified by $\mathrm{E} 3 / \mathrm{E5}$ (top), 5’ $\mathrm{Vb} / \mathrm{E} 514$ (middle) or $\beta 2 \mathrm{~m}$ primer set (bottom). Constructs FLc and G345 are illustrated in Figure 1. $\varnothing$, mock transfection. doi:10.1371/journal.pone.0053469.g002

addition, splicing enhancers and silencers are found within and around G-rich regions (Table 2). Site-directed mutagenesis of Grepeat motifs was studied to determine their roles in HAS1 splicing. Mutagenized sequences for each motif are shown in Figure 3. Splicing profiles driven by various mutagenized G345 constructs are summarized in Figure 4A. Here, we show that Grepeat motifs in HASl intron 3 play an important role in preventing exon 4 skipping. When all 28 G-repeat motifs were disrupted (G345/G1-28 m), the dominant splicing pattern (HAS1FL) was abolished, but splicing to generate HAS IVa was retained $(\mathrm{Va}>>\mathrm{FL})$. Less extensive mutagenesis, affecting only G1-18 (G345/G1-18 m) completely eliminated both FL and Va expression. This was replaced by multiple abnormal spliced products utilizing unconventional cryptic $5^{\prime}$ SS (Figure 4B). The complete loss of $\mathrm{FL}$ and $\mathrm{Va}$ in G345/G1-18 m could potentially be due to altered secondary structure since G345/G1-28 m (all motifs disrupted, including G1-18) still produced HAS1Va. Exon 4 skipping was most pronounced when only G19-28 repeats were mutagenized (G345/G19-28 m), in this case yielding only HASIVa. More refined mutagenesis was studied to define the motifs within G19-28 that are most relevant to prevent exon 4 skipping. An elevated Va:FL ratio was observed among three constructs with mutagenized G19-24, G25-28 and G27-28. The highest Va:FL ratio was produced by G345/G25-28 m, followed by G345/G27-28 m and G345/G19-24 m and was consistent in replicate experiments $(n=5)$. This suggests that they all contribute to the inclusion of exon 4 but at variable degrees and are likely to work additively in this subregion.

Altogether, this analysis showed that sequence modification of critical G- motifs appears to compromise the normal pattern of HAS1 expression by promoting increased exon 4 skipping.

\section{Mutagenesis of G-repeat Motifs in Del1 Construct Promotes HAS1Vb Expression}

Derivatives of dell carrying mutagenized G-repeat motifs were studied in parallel to those of G345 carrying the same mutagenized motifs. Construct dell serves as a model whereby both authentic and alternative 3 'SS in intron 4 are frequently used to form HAS1FL or HAS1Vd (Figure 2B). Since HAS1Vb and HASIVd utilize the same alternative splice site, we asked if manipulation of G-repeat motifs in dell would affect HAS1Vb expression.

Splicing analysis of dell derivatives is shown in Figure 5. Overall, this analysis showed that G-repeat motifs are important for the selection of splicing pathway, consistent with those found in G345 derivatives with the significant exception that all dell derivatives gave rise to increased HAS $1 \mathrm{Vb}$ expression; this did not occur for the G345 derivatives (Figure 4A). In dell/G1-28 m or dell/G19-28 m, HAS1FL expression was almost eliminated and splicing to form HAS $1 \mathrm{Va}$ and HAS $1 \mathrm{Vb}$ became dominant. In dell/G1-18 m, multiple aberrant splicing events predominated over the frequent variants usually detected, in line with those observed in G345/G1-18 m. Splicing was least disturbed in dell/ G19-24 m. Exon 4 skipping driven by dell/G25-28 m was more pronounced than that by dell/G27-28 $\mathrm{m}$, in both cases yielding increased expression of HAS $1 \mathrm{Va}$ and HAS $1 \mathrm{Vb}$ when compared to parental dell. Our study thus demonstrated that aberrant HASIVb splicing could be enhanced by combining genetic manipulation events that lead to increased exon 4 skipping with genetic manipulations that enable increased usage of alternative 3'SS (-59). 


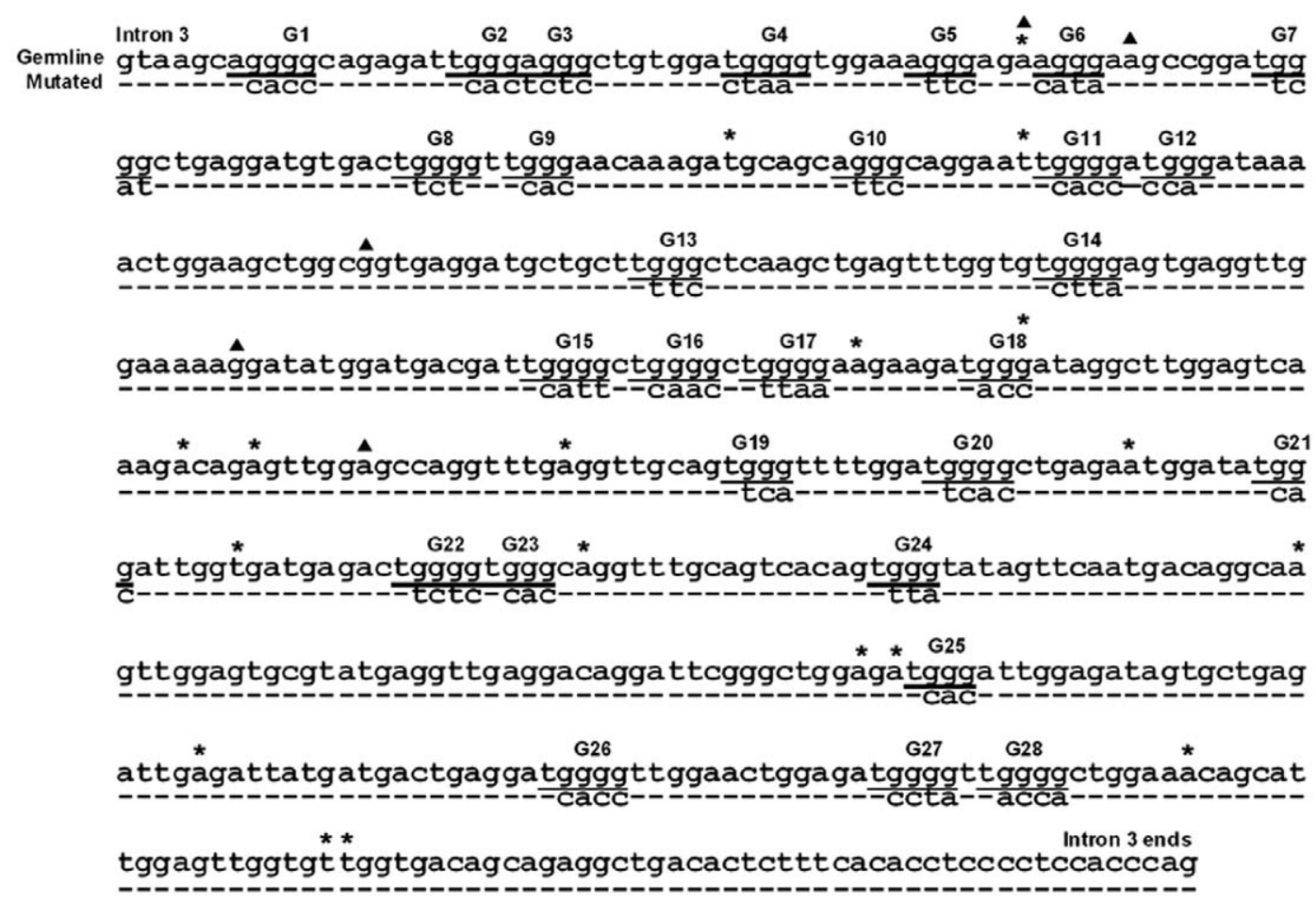

Figure 3. Site-directed mutagenesis of HAS1 intron 3 and recurrent mutations in MM. HAS1 intron 3 sequence is shown (A). (A/U)GGG repeats are underlined and numbered $(\mathrm{G} 1, \ldots, \mathrm{G} 28)$. The mutagenized sequence for each $\mathrm{G}$ motif is shown underneath. Asterisks $(*)$ indicate the positions where recurrent mutations unique to MM PBMC were identified in the $50 \mathrm{MM}$ patients reported here. Triangles $(\mathbf{\Lambda})$ represent recurrent mutations previously identified in 17 patients [21].

doi:10.1371/journal.pone.0053469.g003

\section{Recurrent Genetic Variations in HAS1 Intron 4 and Recurrent Mutations in HAS1 Intron 3 are Frequent in MM Patients}

Since genetic changes in intron 3 and 4 promote changes in aberrant splicing that favor generation of $\mathrm{HAS} 1 \mathrm{Vb}$ (Figure 5), we asked whether genetic variations similar to those created in transfectants are found in genomic HASl of MM patients. Although genetic changes throughout the genome potentially influence local splicing patterns, it was our working hypothesis that mutations distributed within intron 3 may play a significant role. Our initial studies identified 41 recurrent mutations (genetic variations that are shared by 2 or more unrelated patients) in MM patients but not in HD [21]. Of these, 24 recurrent mutations were found in intron 4 and five in intron 3 (marked by $\boldsymbol{\Delta}$ in Figure 3), recurrent in 2-14 of the 17 patients analyzed. In the present study, HAS1 intron 3 was sequenced from a second group of $50 \mathrm{MM}$ patients. For 22/50 patients, 18 recurrent mutations unique to

Table 2. Splicing enhancers (ISE) and silencers (ESS) in the Grich region of HAS1 intron 3.

\begin{tabular}{llcl}
\hline & & & \\
\hline Sequence & \multicolumn{4}{l}{ Type of element References } & Locations ${ }^{1}$ \\
\hline GGGGCTG & ISE (SF1) & {$[28,34]$} & G7, G15, G16, G20, G28 \\
GGGGTTGGGA & ESS & {$[35,36]$} & $\begin{array}{l}\text { G8-9, G11-12, G26, } \\
\text { G27-28 }\end{array}$ \\
GGGATGGGGT & ESS & {$[35]$} & G26, G27 \\
\hline
\end{tabular}

'Location of each G-motif is shown in Figure 3.

doi:10.1371/journal.pone.0053469.t002
MM were identified $(\mathrm{a}>\mathrm{G}=12, \mathrm{t}>\mathrm{C}=5, \mathrm{~g}>\mathrm{A}=1$, marked by $*$ in Figure 3); a significant proportion of these intron 3 mutations were also found in the earlier study but at that point were still presumed to be unique [21]. Individual mutations were recurrent in 2-7 patients. Among these, 17/18 recurrent mutations increased the G-C content of intron 3 and 6/18 either created or disrupted $\mathrm{G}$ runs in intron 3. This demonstrates that mutations frequently occurring in $\mathrm{MM}$ patients are located near those introduced to a construct by in vitro mutagenesis. By extrapolation, these intron 3 mutations in MM patients may contribute to aberrant splicing of HAS1 in malignant cells from patients, where $\mathrm{HAS} 1 \mathrm{Vb}$ is more frequent than HASlVd.

\section{Discussion}

In this work we show that mutations and deletions in introns 3 and 4 of HASl can alter pre-mRNA splicing events to promote aberrant splicing of the type detected in malignant cells from patients with MM. Among splice variants, although HAS1Va (exon 4 skipped) is common, HASı Vb (exon 4 skipped and 59 bp downstream intron 4 retained) appears to be clinically more relevant because in MM, its overexpression correlates with the worst clinical outcome. Since aberrant splicing involves exons 3-45 , it seems likely that frequent mutations in introns 3 and 4 may be involved in the selection of splice sites for pre-mRNA splicing. We utilized HAS1 minigene transfection to evaluate splicing profiles. A newly identified intronic splice variant, HAS1Vd, utilizes an otherwise cryptic splice site in intron 4 to generate a transcript including a segment of intron 4 and encoding a truncated protein. For constructs with unaltered introns 3 and 4, HASIVd transcripts are readily detectable, frequently to the exclusion of $\mathrm{HAS} 1 \mathrm{Vb}$ which utilizes the same intron 4 splice site. In contrast, HAS1Vb is 
A

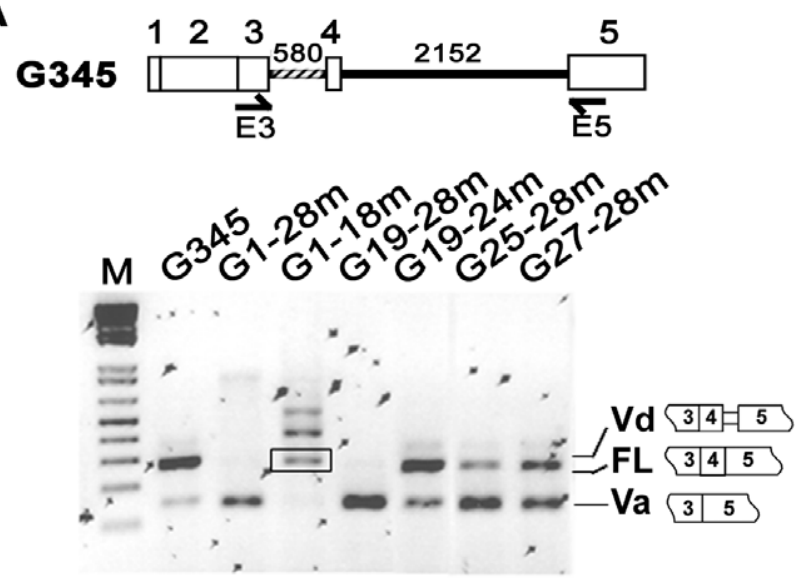

B
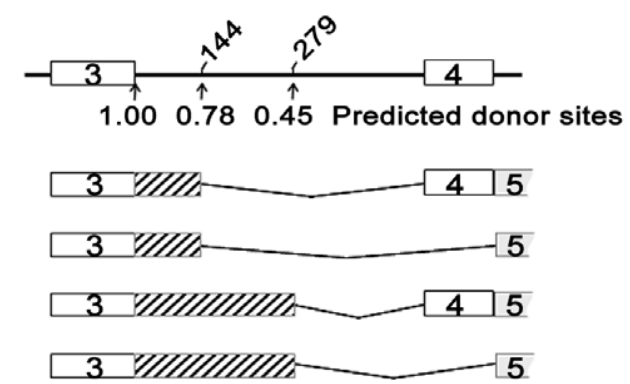

Figure 4. Mutagenesis of G-repeat motifs in HAS1 intron 3 enhances exon 4 skipping. Selected G-repeat motifs in G345 (striped line) were mutagenized according to sequences shown in Figure 3. Splicing profiles driven by various $\mathrm{G} 345$ derivatives were analyzed by RT-PCR using E3/E5 primer set and agarose gel electrophoresis (A). Product in box is not FL as determined by DNA fragment analysis (data not shown). Abnormal HAS1 transcripts driven by G345/G1-18 are summarized in (B). PCR products of G345/G1-18 m transfectants were cloned and spliced junctions were identified by sequencing of subclones. Arrows indicate authentic and cryptic donor sites which located 144 and $279 \mathrm{bp}$ downstream of authentic donor site. The strength of each donor site is determined according to splice site prediction by a neural network (http://www.fruitfly.org/seq_tools/ splice.html).

doi:10.1371/journal.pone.0053469.g004

detected more frequently in patient cells where HAS1Vd is infrequent. For nearly half of MM patients, HAS $1 \mathrm{Vb}$ is expressed in the MM clone at the time of diagnosis [19,21]. For patients lacking HAS1 splice variants at diagnosis, these transcripts were often detected at later stages of disease [19]. Analysis of a series of directed deletions in HAS1 intron 4 showed that splicing of HASIVd could be elevated, but HASIVb remained unaffected, despite their use of the same $3^{\prime}$ splice site in intron 4. Thus, changes in intron 4 alone were insufficient to promote the splicing pattern observed in patients. Combining deletion in intron 4 with mutations in intron 3 however resulted in skipping of exon 4 and promotion of the splicing pattern that leads to a shift from HAS1Vd expression to HAS1Vb expression, the pattern observed in malignant cells from MM patients. To determine the relevance of these genetic changes in vivo, we sequenced intron 3 from genomic DNA of MM PBMC. Consistent with the influence on $\mathrm{HAS} 1 \mathrm{Vb}$ of changes made by site directed mutagenesis, in almost half of MM patients analyzed, we found recurrent mutations in intron 3, some located proximate to $\mathrm{G}$ repeats as well as some that increased the GC content and increased or decreased the number of $\mathrm{G}$ repeats. Previous work has shown that essentially all MM patients analyzed harbored genetic variations in intron 3 and
A

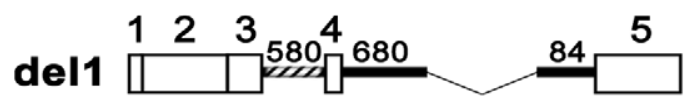

B

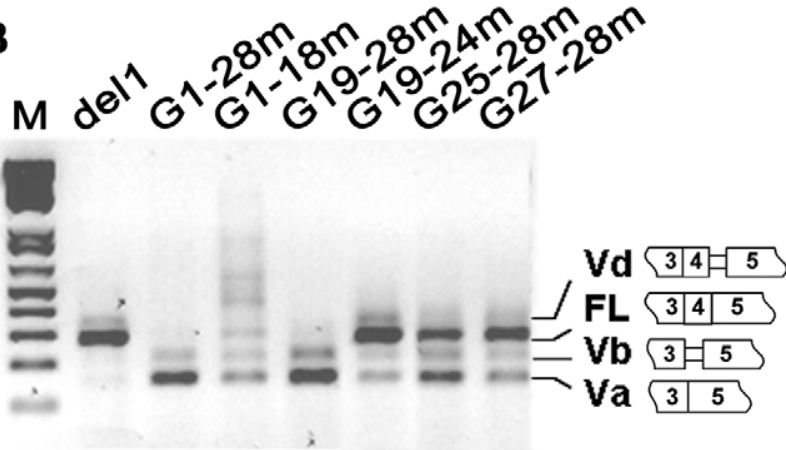

Figure 5. Mutagenesis of G-repeat motifs in del1 promotes HAS1Vb expression. Selected G-repeat motifs in del1 (striped line) were mutagenized according to sequences shown in Figure 3 . Splicing profiles driven by various del 1 derivatives were analyzed by RT-PCR using E3/E5 primer set and products were analyzed by agarose gel electrophoresis.

doi:10.1371/journal.pone.0053469.g005

intron 4 [21]. These observations are consistent with the idea that in MM patients, genetic variations in introns 3 and 4 alter splice site selection resulting in intronic splice variants. Together, these promote use of alternative splice sites to generate intronic splice variants that skip exon 4, operationally resulting in loss of HASIVd splicing and enhanced expression of the clinically relevant HAS $1 \mathrm{Vb}$ variant.

Deletion analysis of intron 4 was aimed at identifying an intronic region that is important for aberrant splicing of HAS1. Mutations previously identified in $\mathrm{MM}$ and $\mathrm{WM}$ are frequent in the two "T" stretches and TTTA repeats of intron 4 [21]. The first $\mathrm{T}$ stretch was removed from deletion construct del5 while both $\mathrm{T}$ stretches were deleted from del4. For del3 and other smaller del constructs, the two T stretches and TTTA repeats were altogether eliminated. Our splicing analysis showed that there was no remarkable change in the splicing profile whether these motifs are present or not, provided that minimum 198 bp sequence (del2) flanking the authentic 3'SS remains undisturbed (Figure 2). While in silico analysis showed that these mutations are important to the formation of $\mathrm{HAS} 1 \mathrm{Vb}$ [21], in vitro splicing analysis did not detect increased expression of HAS $1 \mathrm{Vb}$ even when the usage of relevant alternative 3'SS was increased. Thus, frequent mutations in the common motifs of HASl intron 4 may contribute to aberrant splicing in ways that are beyond the scope of this analysis. Recent epigenetics studies supported the idea that total intronic length could contribute to aberrant splicing via regulation of transcription rate, chromosomal structure and histone modification [24].

G-repeat motifs make up $75 \%$ of intron 3 sequences, thus prompting us to study their influence on HASl splicing. Intronic $\mathrm{G}$ repeats have been shown to modulate splicing in several genes for several species [25-27]. In $\alpha$-globin intron 2, $\mathrm{G}$ triplets acted additively both to enhance splicing and to facilitate recognition of exon-intron borders [28-30]. Likewise, six (A/U)GGG motifs acted additively in IVSB7 of chicken $\beta$-tropomyosin and were essential to spliceosome formation [31]. In human thrombopoietin, intronic $\mathrm{G}$ repeats work in a combinatorial way to control the selection of the proper 3 ' $\mathrm{SS}$; binding to hnRNP H1 is critical for the splicing process as removal of hnRNP $\mathrm{Hl}$ could promote the usage of the cryptic $3^{\prime}$ SS [32]. Our mutagenesis studies showed 
that modification of G-repeat motifs in HAS1 intron 3, especially the last 2-4 motifs of downstream sequence (G25-28 or G27-28), was sufficient to enhance exon 4 skipping (Figure 4). Mutagenesis of intron 3 G-repeat motifs, when combined with an increased usage of alternative 3'SS $(-59)$ caused by intron 4 deletions resulted in an increased HAS1Vb expression (Figure 5). This indicates that the upregulation of aberrant splicing, exemplified here by the expression of HAS1Vb, is influenced by multiple genetic changes in intronic sequences. For HAS $1 \mathrm{Vb}$, this includes enhanced exon 4 skipping and increased usage of alternative 3'SS.

Provocatively, we find that genomic DNA from MM patients harbors novel recurrent mutations in HASl intron 3 and/or intron 4 that are similar to those in the mutagenized HAS1 minigene constructs we introduced to transfectants. In transfectants, the introduction of altered constructs carrying introduced mutations in HAS1 intron 3 and introduced deletions in HAS1 intron 4 promoted a shift to an aberrant splicing pattern already identified as being clinically significant in patients with $\mathrm{MM}$ $[21,33]$. Most MM patients harbor genetic variations in intron 4 [21]. Nearly half of MM patients express HASlVb at diagnosis

\section{References}

1. Kalnina Z, Zayakin P, Silina K, Line A (2005) Alterations of pre-mRNA splicing in cancer. Genes Chromosomes Cancer 42: 342-357.

2. Venables JP (2004) Aberrant and alternative splicing in cancer. Cancer Res 64: 7647-7654.

3. Venables JP (2006) Unbalanced alternative splicing and its significance in cancer. Bioessays 28: 378-386.

4. Buratti E, Baralle M, Baralle FE (2006) Defective splicing, disease and therapy: searching for master checkpoints in exon definition. Nucleic Acids Res 34: 3494 3510 .

5. Pagani F, Baralle FE (2004) Genomic variants in exons and introns: identifying the splicing spoilers. Nat Rev Genet 5: 389-396.

6. Cartegni L, Chew SL, Krainer AR (2002) Listening to silence and understanding nonsense: exonic mutations that affect splicing. Nat Rev Genet 3: 285-298.

7. Colgin LM, Hackmann AF, Monnat RJ Jr (1999) Different somatic and germline HPRT1 mutations promote use of a common, cryptic intron 1 splice site. Mutation in brief no. 259. Online. Hum Mutat 14: 92.

8. Moon SD, Park JH, Kim EM, Kim JH, Han JH, et al. (2005) A Novel IVS2 $1 \mathrm{G}>\mathrm{A}$ mutation causes aberrant splicing of the HRPT2 gene in a family with hyperparathyroidism-jaw tumor syndrome. J Clin Endocrinol Metab 90: 878883.

9. Agrawal S, Pilarski R, Eng C (2005) Different splicing defects lead to differential effects downstream of the lipid and protein phosphatase activities of PTEN. Hum Mol Genet 14: 2459-2468.

10. Auclair J, Busine MP, Navarro C, Ruano E, Montmain G, et al. (2006) Systematic mRNA analysis for the effect of MLH1 and MSH2 missense and silent mutations on aberrant splicing. Hum Mutat 27: 145-154.

11. Lastella P, Surdo NC, Resta N, Guanti G, Stella A (2006) In silico and in vivo splicing analysis of MLH1 and MSH2 missense mutations shows exon- and tissue-specific effects. BMC Genomics 7: 243.

12. Pagenstecher C, Wehner M, Friedl W, Rahner N, Aretz S, et al. (2006) Aberrant splicing in MLH1 and MSH2 due to exonic and intronic variants. Hum Genet 119: 9-22.

13. Durocher F, Labrie Y, Soucy P, Sinilnikova O, Labuda D, et al. (2006) Mutation analysis and characterization of ATR sequence variants in breast cancer cases from high-risk French Canadian breast/ovarian cancer families. BMC Cancer 6: 230.

14. Staalesen V, Falck J, Geisler S, Bartkova J, Borresen-Dale AL, et al. (2004) Alternative splicing and mutation status of CHEK2 in stage III breast cancer. Oncogene 23: 8535-8544.

15. Ludes-Meyers JH, Bednarek AK, Popescu NC, Bedford M, Aldaz CM (2003) WWOX, the common chromosomal fragile site, FRA16D, cancer gene. Cytogenet Genome Res 100: 101-110.

16. Takahashi T, D'Amico D, Chiba I, Buchhagen DL, Minna JD (1990) Identification of intronic point mutations as an alternative mechanism for p53 inactivation in lung cancer. J Clin Invest 86: 363-369.

17. Lamolle G, Marin M, Alvarez-Valin F (2006) Silent mutations in the gene encoding the p53 protein are preferentially located in conserved amino acid positions and splicing enhancers. Mutat Res 600: 102-112.

18. Adamia S, Crainie M, Kriangkum J, Mant MJ, Belch AR, et al. (2003) Abnormal expression of hyaluronan synthases in patients with Waldenstrom's macroglobulimenia. Semin Oncol 30: 165-168.
[19] and as shown here, nearly half harbor recurrent mutations in HAS1 intron 3. Our work suggests that aberrant intronic HAS1 splicing in MM patients relies on intronic HAS1 mutations that are frequent in MM patients but absent from healthy donors. Our previous work, coupled with the molecular analysis reported here, suggests that the splicing regions in introns 3 and/or 4 might represent druggable targets to prevent aberrant HAS1 splicing.

\section{Supporting Information}

\section{Table S1 Expression of HAS1Vb and Vd in MM PBMC.} (DOC)

Table S2 Expression of HAS1Vb and Vd in HD PBMG. (DOC)

\section{Author Contributions}

Conceived and designed the experiments: JK LMP. Performed the experiments: JK AW. Analyzed the data: JK AW. Contributed reagents/ materials/analysis tools: ARB. Wrote the paper: JK ARB LMP.

19. Adamia S, Reiman T, Crainie M, Mant MJ, Belch AR, et al. (2005) Intronic splicing of hyaluronan synthase 1 (HAS1): a biologically relevant indicator of poor outcome in multiple myeloma. Blood 105: 4836-4844

20. Ghosh A, Kuppusamy H, Pilarski LM (2009) Aberrant splice variants of HAS1 (Hyaluronan Synthase 1) multimerize with and modulate normally spliced HAS1 protein: a potential mechanism promoting human cancer. J Biol Chem 284: 18840-18850.

21. Adamia S, Reichert AA, Kuppusamy H, Kriangkum J, Ghosh A, et al. (2008) Inherited and acquired variations in the hyaluronan synthase 1 (HAS1) gene may contribute to disease progression in multiple myeloma and Waldenstrom macroglobulinemia. Blood 112: 5111-5121.

22. Horton RM, Cai ZL, Ho SN, Pease LR (1990) Gene splicing by overlap extension: tailor-made genes using the polymerase chain reaction. Biotechniques 8: $528-535$.

23. Adamia S, Pilarski PM, Belch AR, Pilarski LM (2009) Genetic abnormalities in Waldenstrom's macroglobulinemia. Clin Lymphoma Myeloma 9: 30-32.

24. Luco RF, Allo M, Schor IE, Kornblihtt AR, Misteli T (2011) Epigenetics in alternative pre-mRNA splicing. Cell 144: 16-26.

25. Black DL (2003) Mechanisms of alternative pre-messenger RNA splicing. Annu Rev Biochem 72: 291-336.

26. Nussinov R (1987) (A)GGG(A), (A)CCC(A) and other potential 3' splice signals in primate nuclear pre-mRNA sequences. Biochim Biophys Acta 910: 261-270.

27. Nussinov R (1989) Conserved signals around the $5^{\prime}$ splice sites in eukaryotic nuclear precursor mRNAs: G-runs are frequent in the introns and $\mathrm{C}$ in the exons near both 5' and 3' splice sites. J Biomol Struct Dyn 6: 985-1000.

28. Carlo T, Sterner DA, Berget SM (1996) An intron splicing enhancer containing a G-rich repeat facilitates inclusion of a vertebrate micro-exon. RNA 2: $342-$ 353.

29. Han K, Yeo G, An P, Burge CB, Grabowski PJ (2005) A combinatorial code for splicing silencing: UAGG and GGGG motifs. PLoS Biol 3: e158.

30. McCullough AJ, Berget SM (1997) G triplets located throughout a class of small vertebrate introns enforce intron borders and regulate splice site selection. Mol Cell Biol 17: 4562-4571.

31. Sirand-Pugnet P, Durosay P, Brody E, Marie J (1995) An intronic (A/U)GGG repeat enhances the splicing of an alternative intron of the chicken betatropomyosin pre-mRNA. Nucleic Acids Res 23: 3501-3507.

32. Marcucci R, Baralle FE, Romano M (2007) Complex splicing control of the human Thrombopoietin gene by intronic G runs. Nucleic Acids Res 35: 132 142.

33. Adamia S, Treon SP, Reiman T, Tournilhac O, McQuarrie C, et al. (2005) Potential impact of a single nucleotide polymorphism in the hyaluronan synthase 1 gene in Waldenstrom's macroglobulinemia. Clin Lymphoma 5: 253-256.

34. Carlo T, Sierra R, Berget SM (2000) A 5' splice site-proximal enhancer binds SF1 and activates exon bridging of a microexon. Mol Cell Biol 20: 3988-3995.

35. Wang Z, Rolish ME, Yeo G, Tung V, Mawson M, et al. (2004) Systematic identification and analysis of exonic splicing silencers. Cell 119: 831-845.

36. Bechtel JM, Rajesh P, Ilikchyan I, Deng Y, Mishra PK, et al. (2008) Calculation of splicing potential from the Alternative Splicing Mutation Database. BMC Res Notes 1: 4 . 